\title{
Ética y responsabilidad social de la empresa: su concepción del hombre
}

\section{Ricardo Cuevas Moreno*}

\begin{abstract}
Resumen: El presente trabajo explora la concepción del hombre sobre la cual reposan la Ética de la Empresa (EE) y la Responsabilidad Social de la Empresa (RSE). Según el resultado de esta investigación, dicha noción es una concepción mixtificada del hombre la cual puede funcionar como ideología en el sentido de Marx y Engels (1982). Así la vocación humanística de la EE, la RSE, está confrontada a su carácter de útil de la administración bajo la égida del modo de vida burgués. Esta contradicción se expresa en la práctica que busca el ejercicio de la honestidad, la justicia y la responsabilidad por parte de los líderes de las empresas privadas, frente a los objetivos económicos, morales y sociales de éstas. Así por un lado, existe la aspiración universal y humanista (igualdad, fraternidad, seguridad, justicia y respeto al hombre y su medio ambiente) de la empresa; y por el otro, el primado del trabajo alienado capitalista. Esta contradicción fundamental origina del problema moral de la empresa y los negocios de nuestra época, el cual aparece como irresoluble dentro del contexto del modo de vida capitalista.

Palabras clave: Ética de la Empresa (EE), Responsabilidad Social Empresarial (RSE), hombre, individuo, actor, trabajador del conocimiento, ideología.
\end{abstract}

\section{Ethics and social responsibility in the corporation: its conception of men}

\begin{abstract}
This paper explores the human conception in which corporate social responsibility (RSE) and business ethics (EE) lay. According to the result of this research, such notion is a fault conception of the human being that can function as ideology in Marx and Engels (1982; 1846) sense. The human vocation in business ethics, corporate social responsibility and its philosophy is confronted with the useful sense of management under the businessman way of life. This contradiction is seen in practice when the business men are trying to be honest, fair and responsible and at the same time follow economic goals. In one side is the universal and human side (equality, fraternity, security, justice and human right and environment care) of the firm; and in the other side, the domain of the alienated labor in capitalism. This fundamental contradiction originates the moral problem of the enterprise and of businesses now a days, which seems that has no solution within the capitalistic way of life.

Key words: Ethics on Corporations, Corporation's Social Responsibility, man, actor, intellectual (worker on the field of knowledge), ideology
\end{abstract}

Recibido: 28.03.2009

Aceptado: 10.06.2009

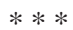

* Universidad Autónoma de Tamaulipas, Tampico, México. E-mail: rcuevasfr@yahoo.fr 


\title{
La administración moral de la empresa y su concepción del hombre
}

\author{
“...el fin por excelencia de la conducta moral, es la \\ sociedad política o la patria, pero la patria concebida como una \\ encarnación parcial de la idea de humanidad." \\ Durkheim (1992; 1902-1903: 68)
}

\section{Introducción}

La administración moderna ha dejado de ser un conjunto de útiles aplicados en la privacidad de la empresa cuyo aprendizaje y práctica conciernen sólo al especialista. El impacto económico, social y cultural de las empresas para el destino de la humanidad suscita una de las preocupaciones mayores del hombre moderno (Bouilloud y Lecuyer 1994: 28) ${ }^{1}$.

Una triada es fundamental en la epistemología de las ciencias de la administración: 1) la integración del conocimiento y el saber en un cuerpo teórico unificado; 2) la pertinencia de las prácticas y su evolución; en fin 3) la enseñanza y aprendizaje de las dos anteriores. Así la instrucción de la administración representa una de los aspectos fundamentales tanto en la creación de especialistas como en la generación del conocimiento nuevo² .

Un problema epistemológico mayor de las ciencias sociales es que la investigación comienza ex post, es decir una vez que los fenómenos se han cristalizado en formas posibles de ser estudiadas. Hubo que esperar que la Ética de la Empresa (EE) y y la Responsabilidad Social de las Empre-

\footnotetext{
${ }^{1}$ Una de las primeras acciones del nuevo presidente de Francia Nicolas Sarkozy es la reunión del 21 de mayo 2007 en el seno de l'Alliance pour la Planète con ocho ONG's a fin de determinar las prioridades en materia de desarrollo sustentable. (http://novethic. fr/ novethic/site/article/index.jsp?id=109346: 23 de mayo 2007)

${ }^{2}$ En 1881 el industrial y financiero Joseph Wharton dona 100 mil dólares para que la Universidad de Pennsylvania fundara un departamento de enseñanza de la Administración. Así nació Wharton School of Commerce and Finance (1881) (George y Álvarez: 2005:76) Algunos años más tarde se fundan las primeras escuelas dedicadas cien por ciento a la enseñanza de la Administración: American Association of Industrial Management (1889), Society for the Promotion of the Science of Management (1911) que se cambia de nombre a Taylor Society; Harvard abre la Harvard Business School (1908) quien tiene entre sus filas a los administradores más ilustres de la época Taylor, Emerson, Morris, Cooke, Day et Going entre otros; a la par de este proceso aparecen los consultantes especializados (Chandler 1988: 517-519).

${ }^{3}$ Se decidió la traducción "Ética de la Empresa” del inglés "Business Ethics”. Este último concepto toma a los negocios y la empresa en bloque como objeto de análisis. En lengua francesa se usa indistintamente "l'éthique de l'entreprise" o "l'éthique des affaires" refiriéndose al mismo objeto de estudio que es la empresa y los negocios. Para evitar confusiones en adelante se dice Ética de la Empresa más acorde con la lengua Española pero teniendo en mente el espíritu del Business Ethics, pues en el presente estudio el objeto de
} 
sas (RSE) tomaran carta de naturalización en la Administración para poder comprender su origen y pertinencia. La adopción de la EE y la RSE por parte de las organizaciones conduce al estudio de los útiles de esa aplicación así como al terreno de la ideología, la disciplina y el poder dentro de la empresa (Cuevas 2005a; 2005b; 2006).

Existen dos ideas comúnmente aceptadas sobre la administración moral de la organización. La primera establece que la EE y la RSE son un útil para mejor administrar la empresa bajo una perspectiva humanista y de cuidado del medio ambiente. La segunda, que al ser un útil de la administración, la EE y la RSE deben servir en la toma de decisiones morales y eficaces para las decisiones operacionales y sobre todo las decisiones estratégicas (Hill y Jones 2000). Además la EE y la RSE deben integrarse a la estrategia de la empresa convirtiéndose de esta forma en guía para la acción e imagen de la empresa.

No obstante, esas dos ideas centrales para la administración moral de la empresa, su concepción del hombre se da por supuesta sin ser explicitada ni explicada. Por tal razón es necesario preguntarse ¿sobre qué concepción del hombre y la sociedad reposan la EE y la RSE? ¿Cuáles son los principales momentos del proceso para que esas nociones tomen forma en el discurso de la administración moral de la empresa? El objetivo del presente trabajo es responder a estas cuestiones.

Para ese propósito la siguiente delimitación es necesaria. La noción de Hombre e Individuo es el resultado de un proceso secular que arranca durante el Renacimiento y se cristaliza en los siglos XVII y XVIII en Europa; esto ocurre en las ciencias de la naturaleza pero sobre todo con la Filosofía Moral y la Economía Política. Dicho proceso toma forma acabada al comienzo del siglo XIX (Foucault 2001; 1966). Por eso más que hacer una genealogía de este proceso para la Administración -tarea que supera con mucho este artículo- aquí se describen los principales momentos de asimilación y transformación de la concepción del hombre por parte de la Economía y la Administración; así como su adopción por parte de la EE y la RSE.

Esa finalidad se cumple estudiando en la primera parte la institucionalización de la EE y la RSE; esto a través de su origen histórico, lo cual conduce a la cultura de la empresa o ideología en donde el individuo es el elemento crucial. Por eso en la segunda parte, se analiza la concepción del hombre que subyace en la administración moral de la empresa a través de las categorías de individuo, actor y trabajador de conocimiento.

En la presente investigación se usa el método de la dialéctica materialista, pues se trata de ver el movimiento de la realidad en sus distintas facetas dentro de su especificidad histórica. En este sentido, la evolución

análisis es la empresa tanto en sus relaciones internas como externas. En estos momentos existe la tendencia hablar simplemente de RSE pues esta siendo la adopción del Desarrollo Sustentable por parte de las empresas contiene lo que se conoce como ética de la empresa. 
histórica del capitalismo y la empresa es un supuesto bien que la exposición se centra en la expresión teórica del mismo.

\section{La institucionalización de la administración moral}

En esta parte se expone brevemente los factores que originan la institucionalización de la EE y la RSE. De igual modo se muestra el origen histórico de éstas así como sus objetos de estudio.

El establecimiento de la Tercera Revolución Industrial, la de la informática y las comunicaciones, en el mundo capitalista coincide con la divulgación de la EE, Desarrollo Sustentable (DS) y RSE (Cuevas 2006). En el lapso de veinte años esa triada que puede nombrarse como administración moral de la empresa, se difunde y adopta en todo el mundo capitalista conocido por varios agentes o vehículos: en primer lugar, las empresas estadounidenses, pasando por lo administradores, consejeros y profesores quienes implantan, difunden y promueve la EE, el DS y la RSE. En segundo lugar, un movimiento paralelo por parte de la Organización de la Naciones Unidas (ONU) quien -a través de reuniones como la Conferencia de Estocolmo sobre el ambiente humano (1972), Cumbre Planeta Tierra o Cumbre de Río (1992), Cumbre Mundial para el Desarrollo Sustentable de Johannesburgo (2002)- establece y difunde la doctrina del Desarrollo Sustentable. Esta nueva concepción del desarrollo capitalista, conjuga las dimensiones económicas, sociales y cuidado del medio ambiente en un todo que pretende terminar con la miseria, el hambre, la injusticia, la inequidad y el desempleo en el mundo. La adopción del DS por parte de las empresas deviene en la RSE de nuestros días.

En tercer lugar, las Universidades y las Escuelas de administración ofrecen cursos y desarrollan investigación sobre ese tema. En cuarto lugar, los centros especializados de carácter público y privado. En quinto lugar, los organismos de acreditación internacional sobre la calidad de la enseñanza, la investigación y desarrollo del business ethics: la Association to Advance Collegiate Schools of Business (AACSB), European Quality Improvement System (EQUIS), la American Mold Builders Association (AMBA). Finalmente, la difusión mediante los medios de divulgación científica (libros, revistas, periódicos y sitios Web). Estos seis agentes vienen a constituir a la EE, el DS y la RSE como una doctrina dentro de las ciencias de la administración y su práctica.

\section{El origen de la Ética en los Negocios y la Responsabilidad Social de la Empresa. Su objeto de estudio y acción}

La EE, el DS y la RSE tienen por origen común las transformaciones profundas de la sociedad y la empresa ocurridas durante el último ter- 
cio de siglo pasado. La degradación acelerada del medio ambiente y las crisis económicas; con ello el desempleo y la miseria de la población. El derrumbe del socialismo, el establecimiento del ejercicio de una nueva soberanía a nivel mundial (Hardt y Negri 2000), la Tercera Revolución Industrial y el establecimiento de la sociedad en red (Castells 2001) o sociedad del conocimiento (Drucker 2000) pueden citarse como los hechos más notables. Esos factores han puesto al capitalismo, la empresa y el ejercicio del poder capitalista en un lugar hegemónico sin parangón alguno en su historia. Las instituciones supranacionales Organización Mundial del Comercio (OMC), Fondo Monetario Internacional (FMI), Banco Mundial (BM) y Organización de las Naciones Unidas (ONU) junto con los Estados del capitalismo desarrollado y las empresas trasnacionales constituyen ese tejido que hace posible el ejercicio del bio-poder (Hardt et Negri, 2000) de la sociedad disciplinaria usando la expresión de Foucault (1975).

En este sentido se podría decir que la EE y la RSE son un resultado de esas transformaciones. Especialmente, la Tercera Revolución Industrial cambia de manera radical el patrón de acumulación capitalista integrando la informática y las comunicaciones en los procesos de producción y circulación del capital. Es en esta revolución en la manera de producir, de cambiar y de consumir junto con la consecuente mutación en la estructura y organización de las empresas, donde la EE y RSE tienen su origen material (Cuevas 2006). Dicha transformación trae como consecuencia la necesidad de administrar de una manera distinta a la empresa pero sobre todo al hombre.

Por otro lado, el poder creciente de las empresas a escala mundial, provoca efectos importantes sobre la humanidad y el medio ambiente. Aunado a esto, la falta de un control supranacional sobre las empresas (Persais 2003), los casos de corrupción y la presión de las Organizaciones No Gubernamentales (ONG's) son otros tantos factores que impulsan el establecimiento de la EE, el DS y la RSE ${ }^{4}$.

\footnotetext{
${ }^{4}$ En referencia a esto cuatro hechos de segundo orden merecen ser citados como motivos del surgimiento de la EN, DS y la RSE. El primero es el caso de la asignación discrecional de los precios de la electricidad en EUA durante la década de 1960. El segundo es el escándalo Watergate (17 junio 1972) después de los cuales el Business Ethics se integra como disciplina en los cursos de MBA. El tercer hecho es relatado como sigue: "Esta responsabilidad social adquirió fuerza legal en 1970, cuando la Ley de Protección Ambiental de Estados Unidos de América creó la Environmental Protection Agency (EPA), como organismo autónomo encargado de administrar las leyes federales en materia de medio ambiente.” (MacMahon 2001: 421 en Frederick 2001). Finalmente la aparición de la Federal Sentencing Guidelines al comienzo de la década de 1990 y cuya finalidad es penalizar las malas conductas en los negocios (Pesqueux y Biefnot 2002: 20-21). ${ }^{5}$ Aubert, (en Bouilloud y Lecuyer 1994:119) opone al management du corps le management des coeurs y affectif para subrayar el cambio de registro de la Organización Científica del Trabajo al Movimiento de las Relaciones Humanas. Prolongando ese razonamiento la noción de subjetividad abarcaría el mundo interior del individuo, del cual la intelectualidad, las emociones, la memoria y el aprendizaje son una parte. Esta noción (gestión de la subjetividad) acuñada por Aubert destacaría una característica importante de la administración actual. Sin embargo,
} 
En resumen, la EE y la RSE son una respuesta a la necesidad de administrar las relaciones del modo de producción (en sentido amplio) y las fuerzas productivas emanadas de la Revolución en la informática y las comunicaciones. Dentro de ésta administración, la gestión de la fuerza de trabajo es un factor clave; sobre todo en la gestión del aprendizaje y el comportamiento moral de los individuos ${ }^{5}$ para el cumplimiento de ciclo del capital a través de una mayor productividad, el consenso y la legitimación. En este sentido, la EE y la RSE contienen además de su dimensión de útil de la administración, una dimensión ideológica en el sentido de Marx y Engels (1982) (Cuevas 2005a; 2005b, 2006).

La definición comúnmente aceptada de RSE estable que ésta es la integración voluntaria de las preocupaciones sociales y ecológicas de las empresas respecto a sus actividades y sus copartícipes. (C.C.E. 2001:7) ${ }^{6}$. Así, para Pesqueux y Bienfnot (2002: XIV, 121, 137, 213-237) la RSE es sobre todo una política de respuesta de las empresas a las demandas de sus partícipes y de la sociedad en general.

$\mathrm{Al}$ asumir este compromiso las empresas asumen una mayor presión, pues, a la dimensión económica de la empresa se integra la del cuidado al medio ambiente y la social (triple bottom line). Dentro de esta última, el cumplimiento de los derechos universales del hombre y la justicia social ocupan el primer plano. En un segundo nivel se encuentra la educación, la cultura y el deporte. La RSE en su implementación revela prácticas distintas ligadas al DS: 1) etiquetas ecológicas, comercio justo y marketing ético o responsable, 2) la Inversión Socialmente Responsable (ISR) y 3) la evaluación de las políticas de RSE.

Así los autores franceses inclinados fundar sobre la definición de conceptos y principios todo discurso, adoptan el concepto de responsabilidad, aplicado al individuo o la empresa, como aquél que puede ser llama-

dicha noción se encuentra ya en Marx (1968; 1844 1965b; 1968) al estudiar el trabajo enajenado y la atención que el proceso de la producción de la plusvalía demanda al elemento subjetivo (fuerza de trabajo) de la producción social.

${ }^{5}$ Aubert, (en Bouilloud y Lecuyer 1994:119) opone al management du corps le management des coeurs y affectif para subrayar el cambio de registro de la Organización Científica del Trabajo al Movimiento de las Relaciones Humanas. Prolongando ese razonamiento la noción de subjetividad abarcaría el mundo interior del individuo, del cual la intelectualidad, las emociones, la memoria y el aprendizaje son una parte. Esta noción (gestión de la subjetividad) acuñada por Aubert destacaría una característica importante de la administración actual. Sin embargo, dicha noción se encuentra ya en Marx (1968; 1844 1965b; 1968) al estudiar el trabajo enajenado y la atención que el proceso de la producción de la plusvalía demanda al elemento subjetivo (fuerza de trabajo) de la producción social.

${ }^{6}$ La definición del objeto de estudio de la EN y la RSE es retomada de Cuevas Moreno, R. y Garza Arroyo, A. (2006): "La relación entre la Responsabilidad Social de la Empresa y la Ética de los Negocios: Una solución a partir de la dialéctica materialista”, CLADEA 2006, 41st Annual Assembly, Latin America \& European Union. Opportunities and Challenges, Montpellier Francia, 10, 11, 12 y 13 de Septiembre. 
do para responder por sus actos o las consecuencias del mismo (Pesqueux y Biefnot 2002; Chauveau y Rosé 2003; Laville 2005).

Esto tiene un fundamento filosófico de precisión del concepto. Así Lalande (2002: 926-928) durante la segunda década del siglo pasado, establece la responsabilidad moral sancionada o no por la ley, a reparar el daño que uno ha causado a otro. Sin embargo, entendido así esa noción encuentra la limitante que la RSE es sobre todo una adhesión voluntaria en donde el castigo jurídico por su incumplimiento se encuentra ausente, bien que en su lugar puede existir la sanción moral por parte de los partícipes de la empresa (stakeholders).

Por todo lo anterior, la puesta en práctica de la RSE aseguraría el bienestar general de la humanidad y su futuro. Sin embargo, la experiencia dicta que bajo el modo de producción capitalista ambos finalidades son difícilmente compatibles, al punto de excluirse. La miseria a escala mundial y la persistente destrucción de los ecosistemas son la prueba palmaria de dicha contradicción ${ }^{7}$.

Por su parte la Ética de la Empresa (EE) puede definirse como la disciplina que discierne lo que es bueno, recomendable, correcto en la administración de las empresas capitalistas, desde un punto de vista ético y moral. Por su naturaleza ella debe ser una herramienta de la administración de la empresa. A partir de este punto de vista una definición como la de Hill y Jones (2000:57) inspirada en el pensamiento anglosajón, resulta limitada. Para estos autores el propósito de la EE es suministrar herramientas para tratar la complejidad moral de las decisiones estratégicas más que prescribir lo que es bueno o malo.

No obstante la EE, el DS tienden a constituir un objeto de estudio único en la práctica a través de la $\mathrm{RSE}^{8}$. La certitud de esa aseveración es posible sustentarla tomando en cuenta dos aspectos. Primero, obsérvese los temas tocados por la EN, el DS y la RSE:

\footnotetext{
${ }^{7}$ En efecto, una declinación acelerada de los ecosistemas en el mundo: 50\% de los bosques de la Tierra son explotados, $30 \%$ de los bosques ancianos están convertidos en campos agrícolas y 9\% de las especies de árboles están en proceso de extinción. 2/3 de las tierras agrícolas experimentan un grado de erosión desde los últimos 50 años. 25 mil toneladas de tierra arable desaparecen anualmente. La sobre explotación de la pesca afecta a un $70 \%$ de las especies marinas. $60 \%$ de los grandes ríos ven afectado su funcionamiento a causa de la canalización, las presas y represas artificiales. En cuanto a la biodiversidad, cada año se extingue entre 20000 y 100000 especies. En fin, la creciente contaminación de las aguas, el aire y la tierra provoca el calentamiento climático. Guide to the World Resources 2000-2001/People and Ecosystems- The Fraying Web of Life. Rapport ONU et le World Resources Institute, 2000 (www.wri.org). Citado por Laville (2006: 65-67).

8 "La ética de los negocios se convirtió en negocios y sociedad o responsabilidad social de la empresas” (MacMahon 2001: 418-420 en Frederick 2001).
} 


\begin{tabular}{|c|c|c|}
\hline $\begin{array}{c}\text { ÉTICA DE } \\
\text { LA EMPRESA } \\
(1985)\end{array}$ & $\begin{array}{c}\text { DESARROLLO } \\
\text { SUSTENTABLE } \\
(1972-2008)\end{array}$ & $\begin{array}{c}\text { RESPONSABILIDAD } \\
\text { SOCIAL DE } \\
\text { LA EMPRESA } \\
(1970-2008)\end{array}$ \\
\hline $\begin{array}{c}\text { Comportamiento } \\
\text { Moral del } \\
\text { administrador }\end{array}$ & $\begin{array}{c}\text { Cuidado del } \\
\text { Medio Ambiente } \\
\text { Recursos Humanos }\end{array}$ & $\begin{array}{c}\text { Eficiencia Económica } \\
\text { Empleo }\end{array}$ \\
Recursos & Eficiencia Económica & $\begin{array}{c}\text { Derechos del hombre } \\
\text { Relaciones con } \\
\text { los stakeholders }\end{array}$ \\
& Justicia Social & $\begin{array}{c}\text { Cuidado del } \\
\text { Medio Ambiente }\end{array}$ \\
& Derechos del Hombre & Relaciones con \\
& los stakeholders \\
& lacha contra & Salud \\
& Salud & Cultura \\
\hline
\end{tabular}

Fuente: Elaboración propia

Segundo, la adopción de la EE, el DS y la RSE es posible gracias a la implementación de una serie de útiles como los son las Cartas y Códigos éticos, los Índices de RSE o DS (IRSE), las auditorias éticas, las certificaciones SA 8000, AA 1000, ISO 9000, ISO 18000- y el Informe de RSE o DS (stakeholder report). Así, hacia el 2009 la RSE contiene una dimensión de útil de la administración heredada de la EE y los aportes del DS -Iniciativas, Recomendaciones, Principios de la ONU, la OIT, OCDE y BIT- y una dimensión ideológica y doctrinaria.

Esas tres dimensiones se encuentran en la implementación de la RSE por parte de las empresas. Más allá del cuidado al medio ambiente, la eficacia económica, la justicia social y el respeto de los derechos del hombre, la RSE responde a la necesidad de administrar a los individuos dentro de la organización haciendo énfasis en su comportamiento moral mediante el aprendizaje y la práctica de los valores morales.

\section{La expresión teórica diversa de una misma problemática}

Por su naturaleza doctrinaria la EE y la RSE han suscitado un número importante de acercamientos teóricos ${ }^{9}$. En este sentido, la administra-

\footnotetext{
${ }^{9}$ Estos enfoques han producido una serie de corrientes ligadas a los nombres de ciertos autores y filósofos. Una corriente inspirada en la teoría de Kant (1994) (Farcy 1969) (Moussé 1989, 1993) (Etchegoyen 1990, 1991) (Landier 1991) (Faber 1992). Otro acercamiento que 
ción moral de la empresa acusa una característica propia de una disciplina como la Administración que es un cuerpo teórico heterogéneo de conocimiento y prácticas.

Así los especialistas basan su estudios en alguno(s) de esos acercamientos produciendo aportaciones, al mismo tiempo que creando una serie de discursos dispares entre ellos (Donalson y Dunfee 1994). Por ejemplo Frederick (2001) en una obra compendiosa, muestra la cantidad prolija de temas y acercamientos dentro del Business Ethics en el mundo anglosajón. En un intento para superar el impasse en el Business Ethics ha caído por su propia lógica, Donalson y Dunfee (1994: 252-254) establecen una división metodológica al parecer pertinente. Por un lado, se encuentran los partidarios del acercamiento empírico preocupados por la solución de problemas tales como la dirección, las decisiones, la motivación, el comportamiento ético y el performance financiero, el marketing, estructura de la organización y problemas de adaptación entre otros. Por otro lado, se encuentran los partidarios del acercamiento normativo. Éstos establecen ideas sobre la dimensión moral de los negocios y las prácticas de la administración que no necesariamente existen en la vida cotidiana de las empresas y los negocios (Donalson y Dunfee 1994: $253^{10}$ ).

Así pues, existe una ausencia de acuerdo desde el punto de vista teórico y práctico sobre el objeto de estudio de la RSE. Bien que existen una serie de útiles para la adopción de la RSE, es difícil aún aislar los factores/criterios que la constituyen por ser una categoría cualitativa. En cuanto a su evaluación, las metodologías, los resultados, datos y series estadísticas están lejos de tener un consenso por lo que su uso para la comprobación de hipótesis resulta difícil para los investigadores. Así una investigación importante, como es la relación entre RSE y rendimiento financiero, hasta hoy carece de una resultado categórico (ORSE 2003; Crifo y Ponssard 2008).

La concepción explicita del hombre se encuentra también ausente dentro de la Administración, el DS, la EE y la RSE. Esta noción se filtra a través del respeto a los derechos universales del hombre propuestos por el DS. Tal vez por ello esa concepción se da por supuesta sin cuestionamiento alguno. Esto resulta paradójico pues la administración moral de la empresa al poner en el corazón del análisis a los valores morales pone en primer plano al hombre ${ }^{11}$. No obstante, es posible deducir el contenido de ese concepto a través de las categorías de individuo, actor y trabajador del

\footnotetext{
${ }^{10}$ Frente a este cúmulo abigarrado de obras no existe un acuerdo sobre los objetos de estudio de EE y la RSE. Por tal razón, en los párrafos precedentes se propone una definición de EE y RSE a partir de las categorías totalidad y praxis comprendiendo a la empresa como una totalidad de relaciones económicas, sociales, políticas, culturales, ideológicas y simbólicas (Cuevas y Arroyo 2006).

${ }^{11}$ Los valores que aparecen con más frecuencia en la literatura de la EE son los siguientes: Autonomía, Honestidad, Responsabilidad, Confianza, Innovación, Valor, Dignidad, Orgullo, Fe, Humildad, Justicia, Libertad, Lealtad, Modestia, Prudencia, Respeto, Solidaridad, Transparencia, Temperancia, Tolerancia. son extraídos de los libros de de Blanchard y Peale
} 
conocimiento, por lo que su estudio es la etapa siguiente.

\section{La administración moral y la concepción del hombre}

En esta parte se analiza cómo la EE y la RSE adoptan una concepción del hombre que proviene de la Economía y de la Administración. Esto conduce a una contradicción importante. Por un lado, nuevos paradigmas como la filosofía económica (Leroux, 1995), (Leroux y Marciano 1999) y la ética económica (Kolm 1996), (Marciano 1999), (Sen, 1986; 1999), (Hirischman 1997), (Mahieu, F-R 2001) ponen al individuo o a la persona ${ }^{12}$ con sus cualidades sociales, morales e ideológicas en el corazón del proceso económico y la empresa. Esto tiende a establecer una concepción de hombre más completa que la heredada por la teoría Neoclásica de la Economía.

En la base del Business Ethics y sus adaptaciones en el mundo capitalista subyace a priori una concepción de la sociedad y del hombre. Esas nociones son un supuesto de los especialistas. Sin embargo, la ciencia debe someter a crítica los supuestos y los resultados de los fenómenos. Al tomar esa actitud con la EE se descubre por ejemplo, que en su construcción, el gran olvido es el trabajador de base. Éste es sustituido por las figuras del "hombre en abstracto", del individuo o del actor (Cuevas 2003: 290-292). En todas las doctrinas económicas y las ideologías existe una concepción de la sociedad y del hombre como supuesto ontológico (Leroux 1995) (Marciano 1999). Pero en el caso de la RSE y la EE dichas concepciones al ser la suposición y razón de ser, se pierden de vista dentro de la construcción de la teoría que sustentan.

Existen cuatro momentos importantes en la construcción de la concepción del hombre subyacente en la administración moral de la empresa y los negocios.

\section{Primer momento: la herencia de Smith}

Smith padre de la economía política inglesa, desarrolla una concepción del hombre y la sociedad a partir de la cual estudia la creación de riqueza en el capitalismo. Smith $(1999 ; 1759)$ formula una teoría ética en

(1988); Le Tourneau (2000), Gélinier (1991), Even-Granboulan (1988), Daigne (1991), Claude (1998), Falise (1992) y Etchegoyen $(1990,1991)$ bien que uno los encuentra citados dondequiera por especialistas en EE y la RSE. Su orden no implica ninguna jerarquía ni la lista pretende ser exhaustiva.

${ }^{12}$ En Francia el Personalismo (1930-1950) pretende ser una tercera vía (humanista y espiritual) entre el liberalismo capitalista y el socialismo. Por eso esta doctrina filosófica opone al individuo a la persona. El primero es considerado como un residuo de la sociedad liberal alienante y en crisis. La persona al contrario, goza de todas las potencialidades espirituales. Una versión reciente de esta doctrina aplicada a la Economía y la Administración se encuentra en (Leroux 1995), Leroux et Marciano (1999) y Marciano (1999). 
donde explica el origen de la moral y el mecanismo de simpatía mediante la cual los hombres crean las normas morales y adquieren juicios morales; al hacerlo, Smith concibe una sociedad a-histórica, libre de contradicciones y en donde el optimismo y la armonía se imponen a pesar de los sentimientos de interés y egoísmo de los individuos.

Smith en su Teoría de los sentimientos morales dice que el gusto por la belleza y la virtud impulsa a los individuos a crear la industria movidos por una mano invisible sin quererlo y sin saberlo sirven a los intereses de la sociedad y dan los medios de la multiplicación de la especie. (Smith 1999; 1759: 422, 256-257, 327, 324). El hombre no es meramente interesado o egoísta, éste sacrifica su interés individual por el bienestar de su sociedad. De esta forma, al igual que los filósofos escoceses de su época como Hume (1751), Smith (1999; 1759) postula la idea del hombre como un ser dotado de pasiones, de virtudes, de defectos y de razón. En el interior del hombre se libra ese encuentro de pasiones naturales que junto con la razón originan la moral y la sociedad.

En desmedro de la idea comúnmente aceptada, es esta concepción de la sociedad y del hombre establecida en la Teoría de los sentimientos morales (Smith 1999; 1759) uno de los fundamentos sobre los cuales reposa la célebre Riqueza de las Naciones (Smith 1991; 1776) (Sen 1986, 1999). Dentro de esas obras se encuentran los valores morales de la libertad, la armonía y la justicia virtudes máximas de su pensamiento.

\section{Segundo momento: el Homo oeconomicus y la Administración}

Pero la teoría Marginalista de la economía retoma una concepción parcial del hombre de la Riqueza de las Naciones (Smith 1991; 1776). Según Kosik (1970: 61-66) la Economía Política clásica se transforma "en ciencia” a partir de la noción de sistema natural. Es a partir de la noción de un sistema que funciona de manera libre y autónoma, como es posible conocer las leyes de la economía. Desde esta idea y este concepto, la Economía Política clásica puede interrogarse sobre las leyes del sistema y sobre las cualidades necesarias del hombre para funcionar dentro del orden económico. Para Smith (1999; 1759) (1991; 1776) el hombre tiene las cualidades de interés, egoísmo, humanidad, beneficencia, bondad, libertad, autonomía e independencia. Continuando con el razonamiento de Smith, sería a partir de ese juego contradictorio de pasiones como surge la moral que funda el sistema económico. Sin embargo, Smith (1991; 1776: 358-361) clasifica a la fuerza de trabajo como un elemento más del capital. Esta cosificación de las relaciones de producción analizada por (Marx 1965; 1867: 604-619) y Rubine (1978) se encuentra al origen de la concepción del hombre en la teoría Neoclásica de la Economía. Ésta reduce la concepción del hombre de Smith a las cualidades del interés, del egoísmo y del cálculo; despojadas de ese gusto por la belleza y el lujo que impulsa a los hombres a desarrollar 
la industria y que tanto llama la atención de Smith en sus dos obras. En suma, la idea de sistema natural a-histórico, en armonía y una concepción parcial del hombre retomados de Smith son los dos pilares sobre los cuales la categoría de homo oeconomicus es postulada.

L'homme economique entidad racional, calculadora, despojada de toda concepción moral y donde su comportamiento es medido con el rigor de las matemáticas, encuentra así, su expresión más refinada con "la revolución marginalista de la economía” de Walras (1834-1910), Jevons (18351882), Menger (1840-1921) y Pareto (1848-1923) ${ }^{13}$. El homo oeconomicus es un individuo racional que ante sus necesidades y los bienes para satisfacerlas, puede establecer un orden de preferencias con la finalidad de maximizar su utilidad y así tomar la decisión óptima.

Algunas críticas sobre la categoría de homo oeconomicus son sucesivamente las de Menger para quien el economista está imposibilitado de dictaminar si los individuos son racionales, locos o morales cuando eligen sus bienes. Por lo tanto, el economista debe constatar el hecho que el individuo que elige sabe que los bienes le darán satisfacción. Otra crítica es la de Veblen (1908) Keynes (1936) y Simon y March (1999; 1958) para quienes el homo oeconomicus está imposibilitado física e intelectualmente para tener toda la información en la toma de decisiones (Demeulenaere 2003; 1996).

No obstante esas críticas, la noción de homo oeconomicus (individuo) es la concepción del hombre que la Administración y la teoría estadística moderna de la decisión adoptan (March y Simon: 1999:135). Dicha noción del hombre se encuentra en las obras de los fundadores de la administración moderna Taylor (1986; 1911) ${ }^{14}$ y Fayol (1981; 1916). En efecto, las obras de Taylor y Fayol se ocupan de elevar la productividad y la eficiencia en la empresa, pero lo hacen modificando involuntariamente la concepción del hombre heredada por la teoría Neoclásica o Marginalismo. Ambos consideran ciertas cualidades sociales y morales en los individuos que deben aprender la tarea o dirigir la empresa.

Aunque los enfoques de Taylor y Fayol difieren, ambos buscan hacer de la administración un quehacer racional y metódico. Al hacerlo continúan la ruptura con el hommo oeconomicus que podemos encontrar en el

\footnotetext{
13 “Con la utilización de la teoría de la utilidad marginal, la investigación sobre el interés personal fue poco a poco puesta a la maximización de un orden de preferencias coherente bajo la hipótesis de un futuro cierto e información completa” (Blaug, 1982:237).

${ }^{14}$ Ribeill (en Bouilloud y Lecuyer, 1994: 31-50) dedica un artículo sobre la vida y obra de Jean-Gustave Courcelle-Seneuil (1813-1892) quien edita Traité théorique et pratique des entreprise industrielles, commerciales et agricoles, ou Manuel des affaires, Paris Guillaumin, 1855 y que es una obra dedicada a la gestión de las empresas en el sentido moderno. Sin embargo, en la historia convencional de la administración este fundador se encuentra ausente.
} 
“humanismo" y "la bonificación por tarea” de Gantt (1901) y en los fundadores de la psicología industrial Munsterberg (1913) y Scott (1910-1911) ${ }^{15}$

Fayol (1981; 1916: 190, 193) por su parte, establece una serie de valores morales que el administrador debe tener para la administración de la empresa y su éxito. Al propio tiempo indica dieciséis responsabilidades para éste entre las cuales encontramos los valores morales de responsabilidad, armonía y justicia.

Taylor $(1986 ; 1911)$ a todo lo largo de su obra principal observa los aspectos sociales, de actitud y de motivación así como las relaciones entre los administradores y los obreros. Para este autor la esencia del management científico consiste en una cierta filosofía derivada de los cuatro principios básicos de la administración ${ }^{16}$.

La ruptura con la concepción limitada del homo oeconomicus continua con algunos autores nombrados por George y Álvarez (2005) como los filósofos de la administración: Sheldon (1923), Mayo (1933), Follett (1930), Mooney (1931), Chester (1938), Burnham (1941). Bien que estos creadores hacen entrar en la administración los valores, los juicios de valor, la importancia del trabajo en equipo, la responsabilidad social del administrador y de la administración; de entre ellos Mayo (1933), con su obra The Human Problems of an Industrializad Civilization, funda un nueva corriente del pensamiento administrativo.

El movimiento de las relaciones humanas surge como reacción a la práctica parcial del pensamiento de Taylor. Los experimentos de Hawthorne perteneciente a la Western Electric en EUA (1924-1933) hacen pensar a los investigadores de aquellos días que dichas “...experiencias demuestran de manera espectacular y definitiva la importancia de las actitudes y los sentimientos de los empleados en el aumento de la productividad.” (Roethlisberger, 1941 citado por Bouilloud y Lecuyer, 1994: 106). Una investigación reciente defiende la idea que entre los resultados de las experiencias de Hawthorne y la interpretación de los mismos existe una marcada diferencia. Pues, en realidad el aumento a la productividad se debió a medidas "taylorianas": pausas, incentivos financieros, disciplina y cambios coyunturales. (Bouilloud y Lecuyer, 1994: 93, 108 116-117).

\footnotetext{
${ }^{15}$ Por ejemplo, Gantt (1901) piensa que la preocupación por el obrero y su moral junto con el elemento humano es uno de los aspectos más importantes de la administración (George y Álvarez, 2005:91-95). Por su parte, Munsterberg (1913) y Scott (1910-1911) consideran la importancia de la mente humana, las condiciones psicológicas y la motivación para la eficiencia industrial. El primero enfocando su análisis sobre las actividades y el segundo sobre el administrador.

16 "Primero, el desarrollo de una verdadera ciencia. Segundo, la selección científica de los trabajadores. Tercero, su formación y adiestramiento científicos. Cuarto, cooperación estrecha y cordial entre el management y los trabajadores.” (Taylor 1911: 117)
} 
Empero, esos esfuerzos de ruptura con el homo oeconomicus no trastocan directamente la categoría clave de la concepción del hombre del Marginalismo que es la racionalidad. La obra de March y Simon (1958; 1999) representa un enorme esfuerzo de síntesis de las dos principales corrientes del pensamiento administrativo (el taylorismo y el movimiento de las relaciones humanas). Al propio tiempo Organizations (March y Simon, 1958; 1999) constituye, a partir de la crítica del homo oeconomicus, una nueva teoría de empresa y las decisiones a partir de una crítica a la racionalidad del individuo.

\section{Tercer momento: la racionalidad limitada del individuo, el actor y el trabajador del conocimiento}

Organizations (March y Simon 1999; 1958: VIII; 21) pretende completar y superar los límites de la teoría nacida de la administración científica (Taylor, 1911), la teoría de "la departamentalización” de Gulick y Urwick (1937), Haldane (1923), Fayol (1930), Mooney y Reiley (1939), Urwick (1943) y del movimiento de las relaciones humanas. Este último, encabezado por Mayo (1933), desarrolla un acercamiento psico-sociológico mientras que la primera profesa un acercamiento racionalista.

La realidad es demasiado compleja para ser tratada en detalle, por lo que se hace necesaria una teoría que sintetice los principales avances de las teorías arriba mencionadas (March y Simon 1999; 1958: 147). En este quehacer existe la necesidad de articular de una forma nueva la concepción del hombre heredada de la teoría Marginalista. Para March y Simon (1999; 1958:12-33) los partidarios de Taylor ven al hombre como una entidad fisiológica capaz de ser disciplinada y adiestrada para realizar la tarea con eficiencia y sólo de manera secundaria admite las cuestiones psicológicas y morales. Al contrario, la escuela de las relaciones humanas, centra su atención en las motivaciones y el trabajo en equipo dejando de lado la otra dimensión.

En el corazón de la solución propuesta por March y Simon (1999; 1958: XI, 138, 185, 197) se encuentra el célebre principio de racionalidad limitada el cual nace de integrar la racionalidad-cognitiva y las aptitudes a la concepción del individuo del marginalismo. Este principio se puede enunciar diciendo que: dada la complejidad de la realidad y el hecho que el hombre es incapaz de aprehender racionalmente (cognitivamente) y en aptitudes toda la información generada por ésta, éste no actúa buscando "la optimalidad" sino que una vez alcanzando "la solución satisfaciente" su búsqueda se detiene.

Así el proceso de toma de decisiones y de innovación se lleva a cabo a través de esquemas de acción. Éstos están constituidos por el conocimiento y la experiencia. Los esquemas forman programas de acción, éstos a su vez constituyen repertorios, los cuales se ponen en acción a partir 
de estímulos recibidos por la empresa para resolver problemas y tomar decisiones.

Organisations (March y Simon, 1999; 1958) representa otro paso mayor en la concepción del hombre para la Administración. Éste debe ser comprendido como una ser fisiológico, con motivaciones, aptitudes y una capacidad racional limitada (cognitividad limitada). A partir de esta última categoría March y Simon crean una nueva explicación del origen de la organización (su estructura y su funcionamiento) y una nueva teoría de la toma de decisiones.

En este sentido March y Simon (1999; 1958) realizan un desplazamiento de gran alcance dentro de la teoría de la empresa. Después de Organisations (March y Simon, 1999; 1958) son la racionalidad y aptitudes limitadas, las motivaciones, la capacidad cognitiva, el saber y la experiencia, el centro de análisis del hombre en la empresa.

La teoría de la acción retoma el concepto de racionalidad limitada para explicar la dialéctica entre l'acteur et le système (Crozier y Friedberg, 1977). Es el actor provisto de estrategias individuales y el mecanismo integrador del juego los que hacen posible el equilibrio entre la libertad individual y las presiones en las empresas. En su accionar el actor busca satisfacerse con el mejor provecho eligiendo las estrategias adecuadas; al hacerlo éste reproduce el sistema del cual forma parte. La Empresa del tercer tipo (Archier y Sérieyx, 1984) retoma la noción de “actor” pero además concebido con sus cualidades de inteligencia y capacidades creativas.

La emergencia de la economía de la informatización y las comunicaciones, pondrá nuevamente en el centro del análisis al individuo pero como trabajador del saber (Drucker, 2002). El cambio fundamental de esta época no se encuentra en la innovación tecnológica o el cambio organizacional, sino en la manera de concebir y aplicar el conocimiento al trabajo. La producción depende del trabajador del conocimiento más que de la tecnología y la organización aunque aquélla las supone ${ }^{17}$.

\footnotetext{
${ }^{17}$ Drucker (2002) al remarcar con justeza este cambio crucial provocado por la Tercera Revolución industrial, confunde la serie de capacidades intelectuales y físicas que definen a la fuerza de trabajo con los medios de producción (Marx 1965b). Para Drucker esas capacidades son "los medios de producción" del trabajador del conocimiento "su propiedad" o “su capital”. Para Drucker (2002: 78-80) el trabajador del conocimiento por antonomasia es el hombre culto. Otro trabajador del conocimiento relevante para Drucker es el gerente o ejecutivo. Pero él olvida que el trabajador de conocimiento debe enajenar sus medios de producción al capital que lo emplea como antes lo hacia el trabajador manual del siglo XIX. Drucker al establecer la diferencia en el uso del conocimiento y el saber deja de lado la naturaleza del modo de producción capitalista cuya relación básica es la compra-venta de fuerza de trabajo, bien que la capacidad que a la empresa capitalista le interese sea la manipulación virtual de símbolos por parte del empleado.
} 
Ahora se está en condiciones de explicar cómo esa concepción del hombre (individuo) que nace de una ruptura -a veces voluntaria y otras involuntaria- con el paradigma neoclásico de la economía, subyace en la EE y la RSE.

\section{Cuarto momento: adopción de la concepción renovada del hombre por parte de la EE y la RSE}

Es evidente que por sus alcances la EE y la RSE requieren una concepción del hombre distinta a la heredada por el paradigma neoclásico de la economía. Un individuo racional que posee la información completa en la toma de decisiones -si existiera- jamás se confrontaría a un dilema moral ni le interesaría necesariamente la dimensión social de las acciones económicas y administrativas. Al contrario, un individuo poseyendo una racionalidad limitada admitiría la dimensión moral y social de sus acciones dentro de la empresa.

La categoría de racionalidad limitada pude servir para comprender cómo la EE y la RSE logran esta adaptación en su lógica interna. En efecto, para la EE una de las aportaciones centrales de las teorías de la organización en su construcción (Cuevas, 2005:103) es la teoría de los Costos de Transacción de O.E. Williamson $(1979,1994)$. El concepto de racionalidad limitada es uno de los supuestos epistemológicos fundamentales de esa teoría (Koening, 1999:147). Es por esa limitación racional-cognitiva que los contratos son incompletos y específicos; su práctica hace posible la coordinación de la producción y su continuación en un medio económico inestable e incierto.

O.E. Williamson $(1979 ; 1994)$ define la empresa como una red centralizada y regulada de contratos específicos. Esta concepción adoptada por la EE hace entrar al propio tiempo la noción de racionalidad limitada y de individuo acuñada por March y Simon (1999; 1958). En consecuencia, la aplicación de la EE permitiría ser una guía y una salvaguarda de las deficiencias de la condición racional-cognitiva limitada del hombre en su comportamiento moral en la empresa ${ }^{18}$.

Por su parte, la RSE toma como marco teórico la teoría de los participantes de la empresa (stakeholder theory). Esta teoría tiende a imponerse en los hechos y los discursos mediante el mimetismo de las políticas de RSE, al punto de tomar aspectos de una ideología (Pesqueux y Bienfnot 2002: 160). Sin embargo, dicho predominio reposa sobre una falta de acuerdo sobre las categorías básicas de esa teoría (Ibid) (D’Humières et. al. 2005).

\footnotetext{
${ }^{18}$ Otro nexo posible de la EN y la RSE con las teorías contractuales de la firma y que además acepta la categoría de racionalidad limitada es la teoría de las convenciones. Al contrario, la teoría de los recursos niega la racionalidad limitada pero establece un nexo entre la RSE como carta mayor de la estrategia (Ver, Persais 2002).
} 
Así por ejemplo, después de la primera formulación de esta teoría de puño del filósofo norteamericano Freeman (1984) una pléyade de trabajos en lengua inglesa se han avocado a definir qué se debe entender por stakeholders, qué tipología de éstos sería pertinente y cuál es la naturaleza de las relaciones participantes-empresa (Chauveau y Rosé 2003:285) ${ }^{19}$.

Al contrario, existe un acuerdo tácito sobre los supuestos de la teoría. 1) La empresa tiene relaciones con distintos grupos al interior y exterior quienes son afectados por sus decisiones; 2 ) la teoría trata de comprender la naturaleza de esas relaciones en términos de procesos y resultados; 3) los intereses de los stakeholders tienen un valor intrínseco; 4) la teoría se interesa por la toma de decisiones (Pesqueux y Bienfnot 2002: 183).

A la luz de la evolución de la teoría de los participantes es de notar que la intención de Freedman (1999 citado por Chauveau y Rosé 2003:283) es la de proporcionar a los administradores políticas cuya eficacia pueda ser evaluada frente a las estrategias de los participantes de la empresa. Al propio tiempo existe una intención ética y social pues existe un aumento de las exigencias éticas que pesan sobre la organización y el individuo junto con el aumento de la concurrencia. De esta forma la teoría de los participantes de la empresa se desarrolla en una vertiente empírica y otra normativa con una tendencia a integrar una sola teoría convergente (Jones y Wicks 1999).

Según el autor de estas líneas, la teoría de los participantes de la empresa resulta pertinente para la RSE por las siguientes razones: 1) A través de los "participantes" se toman en cuenta el juego de relaciones internas y externas de la empresa -en sus dimensiones económica, social y de desarrollo sustentable-, su naturaleza y consecuencias. 2) Al contener un fundamento empírico, normativo y ético, permite el establecimiento de políticas posibles de ser puestas en práctica y ser evaluadas (i.e. D’Humières et. al. 2005: 303-317; La Ville 2006). 3) Por tanto, tomando en cuenta la racionalidad limitada de los participantes de la empresa, resulta un útil para las decisiones estratégicas del individuo, el actor o el trabajador del conocimiento.

\section{Ideología y razón: una insuperable contradicción en la administración moral o Business Ethics}

La evolución del pensamiento económico, administrativo, de la EE y de la RSE pone en el corazón de la gestión de la empresa y el proceso

\footnotetext{
${ }^{19}$ Entre los trabajos más citados en la constitución de la stakeholder theory, se encuentran a Freeman (1984); (Donalson y Preston 1995); (Jones y Wicks 1999); (Jones 1995) (Mercier 1999) bien que March y Simon desde 1958 (1999: 88-92) “establecen cinco grupos mayores” como participantes de la empresa: empleados, accionarios, proveedores, distribuidores y consumidores.
} 
económico una concepción ampliada del hombre. Sin embargo, la EE y la RSE son temas dirigidos a la gerencia. La administración moral de la empresa al ser un útil para mejor conducir a las empresas debe dedicarse principalmente al gerente o al administrador. Por eso el punto clave del análisis para la EE y la RSE no es "el hombre" sino "el individuo" o "el actor" (Cuevas 2003: 290-292), pues éste debe ponerse en acción y tomar decisiones de acuerdo a los objetivos económicos, sociales y morales impuestos por la gerencia.

Este proceso puede ser interpretado de dos maneras distintas. La primera consideraría que a partir de las aportaciones de "los filósofos de la administración”, el movimiento de las relaciones humanas, pero sobre todo, la contribución de March y Simon (1999; 1958), avanza hacia una concepción del hombre total. Al contrario, la segunda interpretación diría que en la teoría de las relaciones humanas y la administración científica aún se distingue en filigrana al hombre (individuo) como sujeto de las relaciones sociales de la producción base de toda empresa capitalista y de la economía; pero con Organisations (March y Simon 1999; 1958) esa perspectiva de análisis es sacrificada en beneficio de la dimensión cognitiva, las motivaciones, el saber, la experiencia y una racionalidad más próxima a la realidad concreta. Esa tendencia se reproduce con las categorías de actor y trabajador del conocimiento. Todos esos conceptos conciben en el fondo al individuo con racionalidad limitada siendo éste o el equipo la unidad de análisis. Al contrario, el análisis por la vía de las relaciones sociales de producción al tener como fundamento las relaciones de propiedad, (Marx y Engels 1982; 1846: 1056-1057; 1085-1090) lleva ineludiblemente al problema del poder, la autoridad, la disciplina y la ideología en la empresa en donde el hombre es el personaje principal.

Justamente para comprender la función económica, social y moral de la EE ha sido necesario someter a crítica sus supuestos, su problemática y sus consecuencias prácticas. Un resultado importante de ese programa de investigación -y que concierne también a la RSE- es que la aplicación de una EE conduce necesariamente a la autoridad, la disciplina y al poder en la empresa. Al seguir ese camino se llega indefectiblemente al terreno de la ideología ${ }^{20}$ y la doctrina. Más allá de ser un útil de la administración la EE contiene una dimensión ideológica cuya función es la reproducción de la condiciones económicas, sociales, políticas y simbólicas dentro de la empresa (Cuevas 2005a, 2005b, 2006, 2007).

\footnotetext{
${ }^{20}$ De acuerdo con Marx y Engels (1982; 1846) se entiende por ideología la forma (representación) que adquieren las relaciones sociales de propiedad, de producción y de poder en la sociedad dividida en clases. La ideología toma la apariencia de ciencia y se presenta como común a todos. Al ocultar las relaciones que la fundamentan la ideología es una falsa conciencia con apariencia de verdad que perpetua las relaciones económicas y sociales de explotación y dominio de una clase sobre otra. En la práctica la ideología impone las ideas y los valores de la clase que domina económicamente e intelectualmente mediante las distintas formas de conciencia social como lo son el Derecho, La Política, La Ética o la Religión. (Cuevas 2005b:100).
} 
En efecto, en el contenido de la EN y la RSE existe una dimensión ideológica y doctrinaria. Ésta es la conciencia y la imagen que el capitalismo y la empresa tienen de sí mismos. La vocación humanista de la administración moral de la empresa se ve confrontada al ejercicio del poder, la autoridad y la disciplina para cumplir los objetivos de la empresa. Ahí no es el individuo en abstracto con racionalidad limitada, el actor o el trabajador del saber sin distinción de rango, quien ejerce el poder y quien ejecuta las ordenes. Al contrario, los trabajadores y empleados en actividad son siempre quienes ejercen y/o son objeto del poder y la autoridad emanada del capital.

En la práctica, el trabajo se desarrolla en el contexto del modo de vida capitalista en donde se reduce al hombre a una actividad circunscrita; es decir la teoría postula una noción hacia un "hombre total” mientras que el trabajo alienado lo circunscribe una actividad delimitada a ciertas funciones y cuyo resultado no le pertenece (Marx 1968; 1844). Por eso esa concepción que pretende ser completa del hombre puede perpetuar las relaciones de propiedad y poder y funcionar, por lo tanto, como una ideología en el sentido de Marx y Engels (1982; 1846).

Marx (1965a; 1857: 235-236) señala con fuerza el carácter imaginario y alejado de la realidad del "cazador", del "pescador" o Robinson Crusoe (Defoe, 1719) individuo aislado y autosuficiente que aparece en la Economía Política clásica (Smith, 1776; Ricardo, 1821). Para Marx ese individuo es una ficción, un reificación (cosificación) del hombre concreto. Según este autor, esa noción olvida que el punto de partida es el hombre que vive y produce en sociedad; que el hombre sólo puede individualizarse en la sociedad; y que además, el "individuo" es un resultado histórico propio del capitalismo. Kosik (1970:62) deriva las consecuencias de esta crítica de Marx pues “...El homo oeconomicus implica la idea de sistema. Él es hombre en tanto parte del sistema y elemento de su funcionamiento, y a ese titulo él debe tener las propiedades indispensables para el funcionamiento del sistema.”

La serie de características de comportamiento del homo oeconomicus son las cualidades que el sistema le solicita para su funcionamiento. Si el hombre económico es racional, egoísta y calculador, es porque existe un sistema donde esas cualidades son necesarias y válidas. Fuera de ese sistema la noción de homo oeconomicus es una abstracción carente de sentido. Pero no es la teoría la que reduce ex nihilo al hombre creando un individuo con una serie de cualidades a priori, es el modo de vida mismo quien efectúa esa reducción.

Lo anterior se repite con las nociones de individuo, actor o trabajador del conocimiento. Ellas alternativamente tratan de completar-se ha visto- la noción lapidaría heredada de las teorías clásica y marginalista de la economía con las motivaciones, las aptitudes, las necesidades, la psique, la moral y el conocimiento. Pero es siempre el individuo o el actor quien debe actuar con una serie de cualidades (autonomía, independencia, cono- 
cimiento experiencia, saber, capacidades, valores morales) para poder obrar dentro de la empresa y el sistema; es siempre el actor o trabajador del conocimiento como administrador o asalariado quien debe obrar como homo faber al servicio del capital.

Así los conceptos devienen en una reificación (cosificación) del hombre real y sus relaciones. Al hacerlo se cree que se estudia al hombre concreto cuando en realidad se analiza una abstracción. Luego, las nociones como individuo, actor y trabajador del conocimiento adquieren la cualidad de ser el parangón de la realidad objetiva consumando el proceso de cosificación. En seguida, al servir para ejercer el poder y modelar el comportamiento moral de los hombres en la empresa, esas nociones pueden funcionar como ideología para mejor administrar la empresa.

Lo anterior se debe a una doble razón: primero, porque sus capacidades físicas y psicológicas son enajenadas en la producción del bien o servicio. Segundo, porque su trabajo se realiza siguiendo los objetivos de la empresa y la ideología impuesta por la gerencia. Así el administrador y el trabajador son "libres” y “autónomos” para identificar su proyecto de vida con el de la empresa. Tercero, porque el resultado de esa enajenación no les pertenece (Marx 1965; 1867).

La investigación sobre las relaciones de autoridad y de poder que la empresa tiene sobre los hombres son sustituidas por los nexos tal como la RSE, la EE y las distintas formas de administración participativa las entienden; es decir, libres de contradicciones, sin especificidad histórica y en armonía. Y cuando los conflictos son admitidos, éstos son despojados de todo su contenido de clase; desplazados y mediatizados hacia los grupos de individuos o participantes de la empresa (stakeholders) con el mismo valor intrínseco, con iguales derechos sin distinción entre ellos.

No obstante son estos conceptos y estas teorías las que son el objeto del proceso de enseñanza-aprendizaje en las escuelas de Administración y se cree además, que son el supuesto para la toma de decisiones de los administradores en las organizaciones. Por un lado, se pretende que el administrador aprenda el bagaje teórico y las herramientas técnicas con la finalidad que éste actúe con eficacia y moral en tanto que individuo total. Pero por el otro lado, el administrador -al igual que el trabajador asalariado- debe hacerlo dentro de una empresa y un modo de vida que lo alinean y lo reducen a una actividad parcial.

Lo anterior tiene una implicación empírica importante. Pues hasta la fecha las empresas capitalistas no han podido resolver de manera práctica la contradicción entre trabajo alienado y florecimiento personal; como tampoco han podido resolver la contradicción entre trabajo alienado por un lado y bienestar social y respeto al medio ambiente por otro. Esta contradicción es impuesta por el modo de vida burgués. La expresión teórica expresa la necesidad de considerar la existencia del 
hombre en todas sus dimensiones mientras que su praxis se desarrolla bajo la égida del capital. Lo anterior es el verdadero problema moral de la EE y la RSE.

De lo anterior se deduce que, sin saberlo, el desarrollo de las teorías de la administración y el Business Ethics han puesto en el centro del análisis dos categorías fundamentales para la teoría del conocimiento de Marx $(1965 a$; 1857; 1968; 1844) totalidad y hombre. Totalidad, pues no se puede estudiar ni conducir a la empresa sin tomar en cuenta el juego de relaciones económicas, sociales, políticas, ideológicas, culturales y simbólicas. El continuum del pensamiento administrativo y la realidad de las empresas demuestran la imperiosa necesidad de aprehender la empresa como una totalidad. El hombre, porque la empresa del nuevo milenio requiere todas sus capacidades objetivas y subjetivas en acción, pero como hombre alienado del capital.

Y aún más, las categorías de totalidad y hombre como categorías epistemológicas para el estudio de la EE y la RSE ponen en evidencia que la Ética y la Moral no son cualidades incluidas del exterior para los negocios y la empresa. Al contrario, la dimensión moral se encuentra orgánicamente unida a los negocios, pero se expresa de una manera negativa debido que éstos se basan en el modo de vida capitalista en donde el interés, el egoísmo y la autoridad de unos hombres sobre otros prima sobre los valores de seguridad, justicia y respeto al medio ambiente. Es de esta forma contradictoria que el destino del hombre y lo humano se expresan en la administración moral. Esta tensión profunda de la EE y la RSE proviene del modo de vida y al parecer es irresoluble dentro de ese contexto.

\section{Conclusiones}

1. En este trabajo se explora la concepción del hombre que subyace en la EE y la RSE. Esa noción es herencia del pensamiento económico y administrativo. Su entrada por parte de la EE y la RSE ocurre mediante la adopción de la Teoría de los Costos de Transacción y la Teoría de los Participantes de la Empresa como marco de referencia en su construcción.

2. La noción actual del hombre en EE y RSE proviene también de una ruptura con la categoría de homo oeconomicus. La concepción actual de hombre para la administración moral se cristaliza en las categorías de individuo, actor y trabajador del conocimiento. Todos esos conceptos conservan en su fundamento la noción de racionalidad limitada.

3. La concepción del individuo ha evolucionado hasta acercarse al concepto de hombre total; es decir, concebido en todas sus dimensiones y relaciones múltiples. Pero los conceptos de individuo, actor y trabajador del conocimiento al convertirse en parangón de la realidad cosifican al hombre y sus relaciones concretas en la empresa. 
4. En consecuencia, esos conceptos podrían funcionar como ideología en el sentido de Marx y Engels (1982; 1846) funcionando de dos maneras: a) ocultando las relaciones de producción y de propiedad privada entre los hombres, por tanto de explotación; b) contribuyendo a reproducir la autoridad, disciplina y poder en la empresa y el modo de vida.

5. Pese a la ruptura con la categoría de homo oeconomicus los conceptos individuo, actor y trabajador del conocimiento siguen cristalizando la reducción que el modo de producción (sistema) hace del hombre real. Pues el modo de vida dicta una serie de cualidades (intelectuales y morales) para que el hombre pueda funcionar en el sistema económico y la empresa en la actualidad.

6. Esta contradicción proviene del modo de vida capitalista fundado en la alineación individual privada de los productos del hombre. Por tal razón persiste el hecho que el trabajo alienado (la ganancia) entra en contradicción con "el florecimiento personal”, los derechos del hombre, el bienestar social y el respeto al medio ambiente. Este es el verdadero problema moral de la empresa y los negocios.

7. En consecuencia, se abre la posibilidad de un programa de investigación de una mayor profundidad y amplitud a fin de apreciar en detalle, la construcción de la concepción del hombre que subyace en la administración moral de la empresa y los negocios. Esto es de capital importancia, pues la empresa determina en gran medida el destino del hombre moderno. 


\section{Bibliografía}

Archier, G. et Sérieyx, H. (1984), L'entreprise du $3^{e}$ type, Editions Seuil, Paris.

Bouilloud, J-P et Lecuyer, B-P. (1994), L'invention de la gestion. Histoire et pratiques, Harmattan, Paris.

CNUED Déclaraction de Rio Sur l'Environnement et le Développement. Rio de Janeiro, Brasil, 3-14 juin 1992, ONU, Obtenido de : http:// www.un.org/french/events/rio92/aconf15126vol1f.htm

Idem (1992), La Action 21, Nations Unies. Développement Durable, Cumbre de Río Obtenido de : http://www.un.org/french/ga/special/sids/agenda21/action0.htm

Chauveau y Rosé, (2003), L'Entreprise responsable : Responsabilité sociale - Ethique, Édtions d’Organisation, Paris.

Claude, J-F. (1998), L'éthique au service du management, Les Éditions LIAISONS, Paris.

Cuevas, Moreno, R. (2003), L'éthique de l'entreprise capitaliste: Essai sur "L'éthique de l'entreprise” en France et la idéologie, Université de Corse Pascal Paoli, Institut d'Administration des Entreprises (IAE), Thèse de Docteur en Sciences de Gestion, France, 8 avril 2003.

Idem (2005a), “La construction de l'éthique de l'entreprise. Première Partie. Les formes à partir de l'adoption de l'éthique”, Revista de Contaduría y Administración. Nueva Época arbitrada, Núm. 216, UNAM.

Idem (2005b), “La construction de l'éthique de l’entreprise. Seconde Partie. Les formes à partir de l'adoption de l'économie par l'éthique de l'entreprise", Revista de Contaduría y Administración. Nueva Época arbitrada, Núm. 217, UNAM, México.

Idem (2006), “La ética de los negocios y la economía de la informatización”, Revista de Contaduría y Administración. Nueva Época arbitrada, Núm. 219 UNAM, México.

Idem (2007), “Las formas de la ética de los negocios: la síntesis o las formas propiamente dichas. La doctrina social de la iglesia cristiana”, Revista de Contaduría y Administración. Nueva Época arbitrada, Núm. 221 UNAM, México.

Cuevas Moreno, R. y Garza Arroyo, A. (2006), “La relación entre la Responsabilidad Social de la Empresa y la Ética de los Negocios: Una solución a partir de la dialéctica materialista”, CLADEA, 41st Annual Assembly, Latin America \& European Union. Opportunities and Challenges, 
Montpellier Francia, 10, 11, 12 y 13 de Septiembre.

De la Bruslerie, H (coordonnateur) (1992) Éthique, Déontologie et Gestion de l'Entreprise, ECONOMICA collection Gestion, Paris.

D’Humières, P. (2005), Le développement durable, Éditions d’Organisation, Paris.

Demeulenaere, P. (2003; 1996), Homo oeconomicus, Presses Universitaires de France, collection Quadrige, Paris.

Donalson y Dunfee, “Toward a unified conception of business ethics: Integrative Social Contracts Theory”, Academy of Management Review, Vol. 19 No. 2 1994, pp, 252-284.

Commission des Communautés Européennes (C.C.E.) (2001), Livre Vert. Promouvoir un cadre européen pour la responsabilité sociale des enterprises, Bruxelles.

Drucker, P. (2002), Escritos fundamentales, T. 1. El Individuo, Editorial Sudamericana, Argentina.

Durkheim, E. (1992 ; 1902-1903), L'éducation morale, PUF, Paris.

Etchegoyen, A. (1991), La valse des Ethiques, Editions François Boudin, Paris.

Idem (1990), Les entreprises ont-elles une âme ? Editions François Bourin, Pairs.

Even-Granboulan, G. (1988), Ethique et Economie. Quelle morale pour l'entreprise et le monde des affaires ? L'Harmattan, Paris.

Faber, E. (1992), Main basse sur la Cité. Ethique et entreprise, éditeur Hachette, France.

Farcy, H (présentateur) (1969) Vers une morale des affaires ? L'Ethique du Responsable commercial, Institut catholique de Paris. Bibliothèque de la Action populaire. Bibliothèque de la Recherche Sociale. SPES.

Fayol, H. (1981), Administración industrial y general, Argentina, Librería “El Ateneo” Editorial, Traducción del francés de Dr. Dimitru, C. Paris.

Foucault, M. (1975) Surveiller et punir. Naissance de la prison, Éditions Gallimard, France.

Idem. (2001 ; 1966), Les Mots et les Choses. Une archéologie des sciences humaines. Editions Gallimard, France.

Frederick, R.E. (2001) La ética en los negocios. Aplicación a problemas 
específicos en las organizaciones de negocios, OXFORD, University Press, Traducción del inglés Carril Villarreal, M. del Pilar. México.

George, C. S. y Álvarez, L. (2005), Historia del pensamiento administrativo, Pearson, Prentice Hall, Traducido del inglés, México.

Gélinier, O. (1991), L'éthique des affaires. Halte à la dérive, Editions du Seuil, Paris.

Habermas, J ; Morale et communication (1986), Flammarion, Traduction et Introduction, par Bouchindhomme, C. Paris.

Hardt, M et Negri, A. (2000), Empire, Exils Editeur, traduit de l'américain par Canal, D-A. Paris.

Hirschman, A. (1997), La morale secrète de l'économiste, Editeur Les Belles Lettres, , Entretiens avec Donzelle, C ; Petrusewicz, M et Rusconi, C ; Traduit de l'italien par Dauzat, P-E . Paris.

Hill, C. y Jones, G. (2000), Administración estratégica un enfoque integrado, McGraw-Hill, Colombia.

Kant, E. (1994), Métaphysique des mœurs, GF, Flammarion, Paris.

Kolm, S-C. (1996), Philosophie de l'Économie, Éditions du SEUIL, Paris.

Kosik, K. (1970) La dialectique du concret, Traduit de l'allemand par Dangeville, R. Maspero, Paris.

Landier, H. (1991), Vers l'entreprise intelligente. Dynamique du changement et mutation du changement, Editions Calmann-Lévy, Paris.

Lalande, A. (1991), Vocabulaire technique et Critique de la Philosophie,

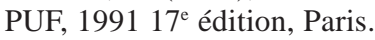

Laville; E. (2006), L'entreprise verte, Village Mundial, France.

Leroux, A. (1995) Retour à l'idéologie. Pour un humanisme de la personne, PUF, Paris.

Leroux, A et Marciano, A (sous la direction) (1999), Traité de philosophie économique, BALISES, Paris.

Marciano, A. (1999), Ethiques de l'économie. Introduction à l'étude des idées économiques, BALISES, Paris.

March, J-G. et Simon, H.-A. (1999), Les Organisations. Problèmes psychosocilogiques, Dunod, Traduit para Rouchy J.-C. et Pruier, G. 2èm édition, Paris. 
Marx, K. (1968 ; 1844) Manuscrits parisiens, Euvres III Philosophie, NRF Gallimard, Paris.

Idem 1965a; 1857), Introduction générale à la critique de l'économie politique, Euvres I Economie, NRF Gallimard, Paris.

Idem (1965b, 1867), Le Capital, livres I, II et III, Euvres I Economie, NRF Gallimard, Paris.

Idem (1982a ; 1845), De l'a abolition de l'État à la constitution de la société humaine. Ad. Feuerbach (Thèse sur Feuerbach), Euvres III Philosophie, NRF Gallimard, Paris.

Marx, K et Engels, F. (1982b ; 1846), L'Idéologie allemande, Euvres III Philosophie, NRF Gallimard, Paris.

Mahieu, F-R. (2001), Ethique Economique. Fondements anthropologiques, L'Harmattan, Paris.

Mercier, S. (1999), L’éthique dans les entreprises, La Découverte, Paris.

Mintzberg, H. (2001 ; 1989), Le management, voyage au centre des organisations, Editions d’Organisation, traduit par Behar, J-M, quatrième édition, Paris.

Morris, T. (2005) Si Aristóteles dirigiera General Motors. Las enseñanzas clásicas para sobrevivir en un mundo competitivo, Editorial Planeta, España.

Moussé, J. (1993) Éthique et entreprises, éditeur Vuibert, Paris.

Idem (1989), Fondements d'une éthique professionnelle, Les Editons D’Organisation, Paris.

Novethic. Fr. Le gouvernement lance le grenelle de l'environnement, France, 23 de mayo 2007 Obtenido de : http://www.novethic.fr/

ONU. (2002), Rapport du Sommet mondial pour le développement durable Johannesburg, Afrique du Sud, 26 août-4 septembre 2002. Obtenido de: http://www.sommetjohannesburg.org/

Idem (1992), Rapport de la Conférence des Nations Unies sur l'environnement et le développement, Rio de Janeiro, 3-14 juin 1992 (publication des Nations Unies, numéro de vente : F.93.I.8 et rectificatifs). Obtenido de : http://www.un.org/french/events/wssd/pages/cnued.html

Persais E. (2003), “Le rapport de développement durable (ou stakeholders’ report) : un outil pour une gouvernance sociétale de l'entreprise ? ", Journée AIMS Développement durable et entreprise, 15 mai, Angers, 2003. 
Pesqueux, Y. et Biefnot, Y. (2002) L'éthique des affaires. Management par les valeurs et responsabilité sociale, Éditions d’Organisation, Paris.

Rubine, I.I. (1978) Essais sur la théorie de la valeur de Marx, Maspero, Paris.

Sen, A. (1986), Ethique et économie, et autres essais, PUF, traduit de l'anglais par Marnat, S. Paris.

Idem (1999, L’économie est une science morale, Paris, Editeur, La Découverte.

Smith, A. (1999), Théorie des sentiments moraux, Traduction par Biziou, M, Gautier, C, Pradeau, J. PUF, Paris.

Idem (1991), Recherche sur la nature et les causes de la richesse des nations, Traduction de Garnier, G. GF. Flammarion, Paris.

Solomon, R.C. (2000), Nuevas reflexiones acerca de las organizaciones de negocios. El éxito basado en la integridad de las personas, Oxford University Press, Traducido del inglés por María del Pilar Carril Villarreal, México.

Koening, G. (coordinateur) (1999), De nouvelles théories pour gérer l'entreprise du XXI siècle, ECONOMICA, Paris.

Taylor, W. F. (1986; 1911), Los principios del management científico, Ediciones Orbis, S.A. Traducción Alicia Arrufat, quinta édition, España.

Williamson, O.E. "Transaction Cost Economics: The Governance of Contractual Relations”, Jounal of Law and Ecomomicx (No. 2, 1979).

Williamson, O.E. (1994), Les institutions de l'économie, InterEditions, Paris. 